\title{
Making sense of low oxygen sensing
}

\author{
Julia Bailey-Serres ${ }^{1}$, Takeshi Fukao ${ }^{1}$, Daniel J. Gibbs ${ }^{2}$, Michael J. Holdsworth ${ }^{2}$, \\ Seung Cho Lee ${ }^{1}$, Francesco Licausi ${ }^{3,4}$, Pierdomenico Perata ${ }^{4}$, \\ Laurentius A.C.J. Voesenek ${ }^{5,6}$ and Joost T. van Dongen ${ }^{3}$
}

\author{
${ }^{1}$ Center for Plant Cell Biology, Department of Botany and Plant Sciences, University of California, Riverside, CA 92521-0124, USA \\ ${ }^{2}$ Division of Plant and Crop Sciences, School of Biosciences, University of Nottingham, Loughborough LE12 5RD, UK \\ ${ }^{3}$ Max Planck Institute of Molecular Plant Physiology, Am Muehlenberg 1, 14476 Potsdam-Golm, Germany \\ ${ }^{4}$ PlantLab, Institute of Life Sciences, Scuola Superiore Sant'Anna, Piazza Martiri della Libertà 33, 56127 Pisa, Italy \\ ${ }^{5}$ Plant Ecophysiology, Institute of Environmental Biology, Utrecht University, Padualaan 8, $3584 \mathrm{CH}$ Utrecht, the Netherlands \\ ${ }^{6}$ Center for Biosystems Genomics, 6708 PB Wageningen, the Netherlands
}

\begin{abstract}
Plant-specific group VII Ethylene Response Factor (ERF) transcription factors have emerged as pivotal regulators of flooding and low oxygen responses. In rice (Oryza sativa), these proteins regulate contrasting strategies of flooding survival. Recent studies on Arabidopsis thaliana group VII ERFs show they are stabilized under hypoxia but destabilized under oxygen-replete conditions via the $\mathrm{N}$-end rule pathway of targeted proteolysis. Oxygendependent sequestration at the plasma membrane maintains at least one of these proteins, RAP2.12, under normoxia. Remarkably, SUB1A, the rice group VII ERF that enables prolonged submergence tolerance, appears to evade oxygen-regulated $\mathrm{N}$-end rule degradation. We propose that the turnover of group VII ERFs is of ecological relevance in wetland species and might be manipulated to improve flood tolerance of crops.
\end{abstract}

\section{Improved crop survival of floods is needed}

Based on conservative expectations of human population growth, the maintenance of international food security will require a doubling of agricultural productivity in the next two decades [1]. This challenge is exacerbated by severe weather events associated with climate change such as floods, which have occurred with increasing frequency across the globe over the past six decades (Figure 1). However, improvement of crop resilience to water extremes can be accomplished by harnessing natural genetic diversity in breeding programs. An example of this is the use of the rice SUBMERGENCE 1A (SUB1A) gene, which confers prolonged tolerance to submergence (see Glossary) [2]. The effective SUB1A-1 allele was isolated from an eastern Indian landrace and has been returned to farmers in high-yielding varieties [3]. This new 'Sub1 rice' promises to help stabilize harvests in rainfed floodplains, which represent $33 \%$ of rice acreage worldwide [4]. The task remains to improve flooding tolerance of other crops. Recent comparative studies within and between species have greatly enhanced our understanding of mechanisms that facilitate survival of distinct flooding regimes (Table 1). With new insights into low oxygen sensing and response mechanisms we are optimistic that effective means to lessen crop devastation by flooding can be extended beyond rice paddy fields.
Oxygen deprivation is a frequent component of flooding stress

A key feature of flooding events is the change in levels of three gases, $\mathrm{O}_{2}, \mathrm{CO}_{2}$ and ethylene, due to a near $10^{4}$ reduction in their diffusion in water relative to air [58]. The flooding of root systems - a condition termed waterlogging - has little or no impact in semi-aquatic species such as rice that constitutively form gas conduits (i.e. aerenchyma) between submerged and aerial organs. However, if plants lack gas conduits or lose oxygen from roots, waterlogging rapidly reduces the oxygen concentration within cells [9-11]. The presence of aerobic microbes in the soil can further exacerbate the stress. When both the root and aerial portions of a plant are whelmed by water -a condition termed submergence - cellular oxygen levels can also decline from normoxia. The degree of oxygen deficiency (hypoxia/anoxia) depends on multiple factors including replenishment of oxygen through photosynthesis, inward diffusion from the water layer and cellular consumption of oxygen through metabolic activity. Severe oxygen deficiency compromises mitochondrial respiration [10,12] and leads to an insufficiency in ATP for energy demanding processes [6,13-15]. However, plants can adjust to this energy crisis through increased substrate level ATP production (Figure 2). This is accomplished by catabolism of soluble sugars and in some species or cell types starch [16]. Typically, the increase in glycolytic flux is coupled with regeneration of $\mathrm{NAD}^{+}$by fermentation of pyruvate to

\footnotetext{
Glossary

Anoxia: absence of oxygen.

Direct oxygen sensing: sensing of oxygen via its molecular interaction with a ligand (i.e. enzyme, protein, chemical compound) that results in an effect of cellular consequence.

Hypoxia: oxygen levels below normoxia; the term 'hypoxic' is often used to describe a situation where molecular oxygen is still present, but its level has significantly decreased below $20.6 \%$; cellular oxygen status may be hypoxic or anoxic dependent upon duration, location and metabolic activity.

Hypoxia-responsive genes: genes with transcripts differentially regulated in response to conditions with a low oxygen component.

Indirect oxygen sensing: sensing of change in homeostasis that is a consequence of oxygen deprivation (i.e. change in ATP, ADP, AMP, other metabolite, $\mathrm{Ca}^{2+}, \mathrm{ROS}, \mathrm{pH}$ ) that results in an effect of cellular consequence. Normoxia: typically $20.6 \%$ oxygen at $1 \mathrm{~atm}$ and $20^{\circ} \mathrm{C}$.

Submergence: waterlogging and partial to complete immersion of aerial system.

Waterlogging: flooding of root system.
} 


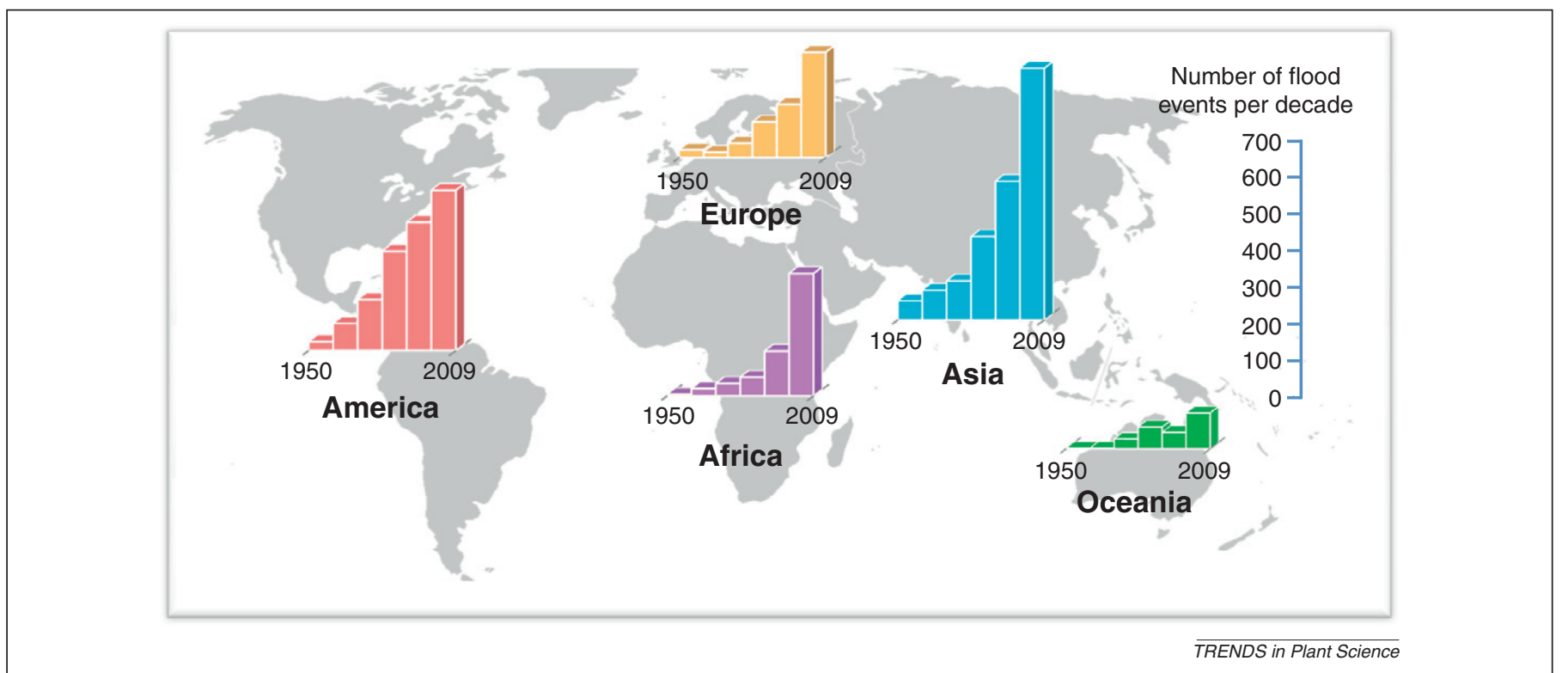

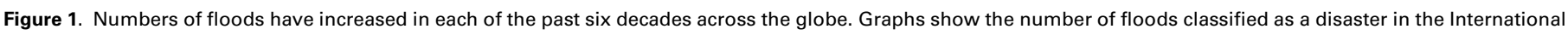
Disaster Database of the University of Louvain, Belgium for the period from 1950 through 2009 by geographical region [93]. Events include river or coastal floods, rapid snow melts, heavy rainfall and other occurrences that caused significant social or economic hardship. Adapted from a Millennium Ecosystem Assessment map (http:// maps.grida.no/go/graphic/number-of-flood-events-by-continent-and-decade-since-1950).

ethanol via pyruvate decarboxylase and alcohol dehydrogenase $(\mathrm{ADH})$. Because ethanol diffuses out of cells into the external milieu, its production depletes the plant's carbon reserves. Therefore, metabolism of pyruvate to alanine provides an alternative, non-detrimental end product of anaerobic metabolism that is observed in a number of species $[17,18]$. This includes the generation of 2-oxoglutarate as a coproduct, which can be further metabolized to succinate, via the TCA cycle enzyme succinate CoA ligase (SCS), thereby providing additional ATP per molecule of sucrose metabolized. To keep these reactions running, the oxidation of NADH in the mitochondrial matrix is guaranteed by reduction of oxaloacetate via the reversed TCA cycle reaction catalyzed by malate dehydrogenase $[19,20]$. The malate produced is probably further converted to fumarate and succinate [21], the latter of which could be exported from hypoxic tissue to the aerated parts of the plant. At least in tubers of potato (Solanum tuberosum), hypoxia stimulates a rearrangement of the mitochondrial respiratory supercomplexes that enhances regeneration of $\mathrm{NAD}^{+}$by the alternative $\mathrm{NAD}(\mathrm{P}) \mathrm{H}$ dehydrogenases [22].

Even though the efficiency of hypoxic ATP production is low compared to aerobic oxidative phosphorylation, it allows cells to survive as long as carbohydrate substrate remains available. Cell death only becomes inevitable when there is insufficient energy for exclusion of protons to the apoplast to prevent membrane depolarization and to maintain a near neutral cytosolic $\mathrm{pH}[6,23,24]$. Avoidance of the severe energy crisis associated with low oxygen stress requires economization of ATP consumption. Means to this end include energy efficient sucrose catabolism through sucrose synthase [25], the preferential use of PPi-dependent enzymes [26], constrained catabolism of storage compounds such as starch, lipid and protein [13], metabolic compartmentalization [27], reduced protein synthesis [28], increased production of heat shock proteins as molecular chaperones [29] and adoption of the $\mathrm{K}^{+}$-gradient to energize membrane transport [30]. Plant survival of waterlogging or submergence also depends on their ability to limit or endure oxidative stress, which occurs during the transition from normoxia to anoxia as well as upon de-submergence [31-33].

\section{Ethylene initiates submergence survival strategies in rice and wetland species}

Recent work has exposed mechanisms of response to submergence that center on growth management. Notable are two antithetical survival strategies displayed by both wild and domesticated species. For example, deepwater rice, cultivated to cope with slowly advancing floods, expends energy reserves in the elongation of internodal regions that are underwater to maintain photosynthetic tissue above the air-water interface [34,35]. Similarly, the wetland dicot Rumex palustris, which is well adapted to shallow but prolonged floods, reorients and extends petioles to elevate leaves above the surface of floodwaters [6,7]. However, this 'submergence escape' strategy is unsuccessful if energy reserves are exhausted before escape of the deluge. In wetland species capable of surviving transient floods (e.g. Rumex acetosa) [36] and submergence tolerant Sub1 rice [37], a 'quiescence strategy' minimizes energy expenditures for growth until de-submergence.

The genetic determinants and hormonal signaling pathways that underlie the two flooding survival strategies have been identified. In rice, both strategies utilize the phytohormone ethylene and ethylene response factor (ERF) transcription factors. Combined physiological and molecular dissection of submergence responses in rice and $R$. palustris has yielded a model in which a buildup of ethylene in submerged organs initiates a hormonal signaling cascade that reduces the antagonism between abscisic 
Table 1. Factors that contribute to survival of flooding or oxygen deprivation

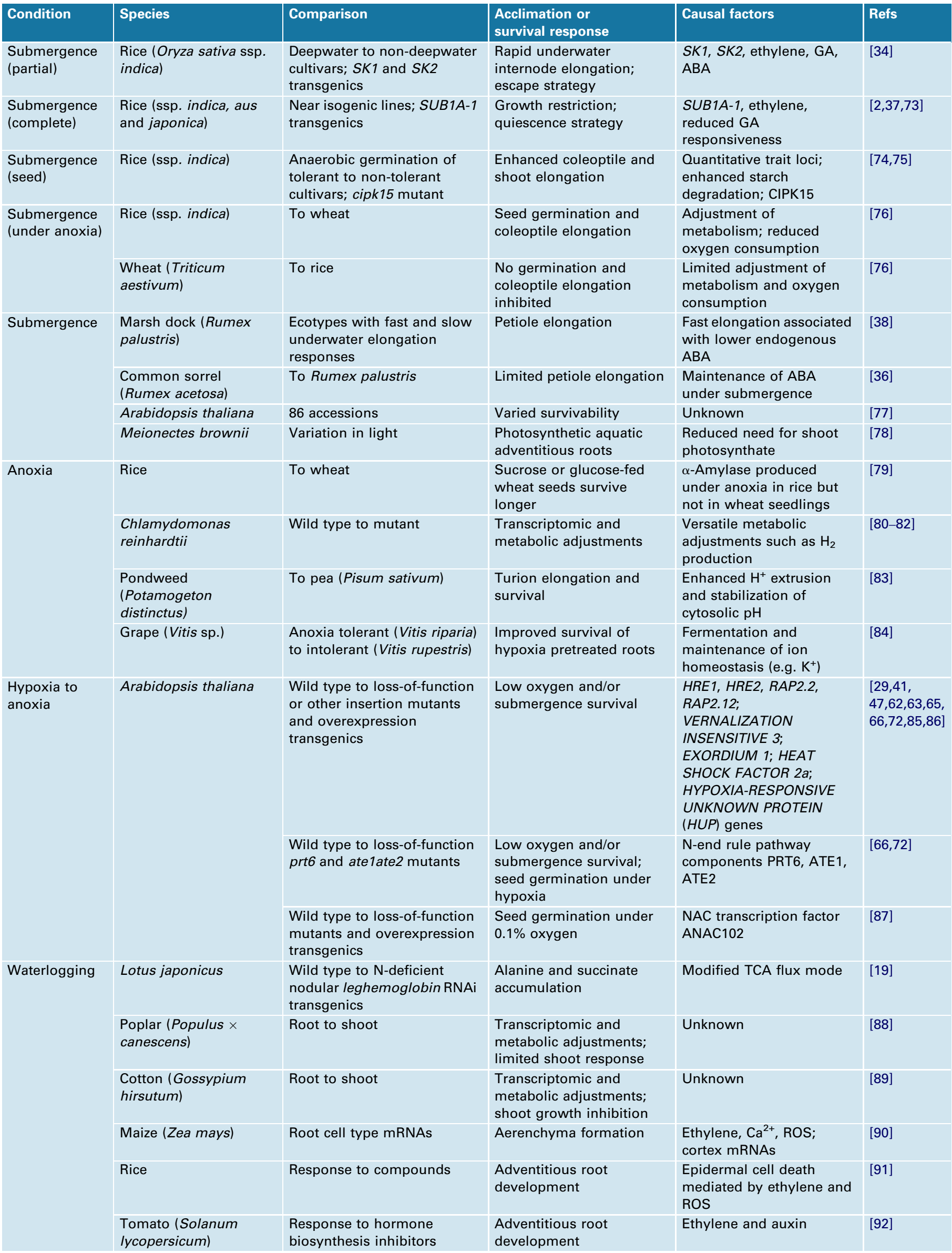




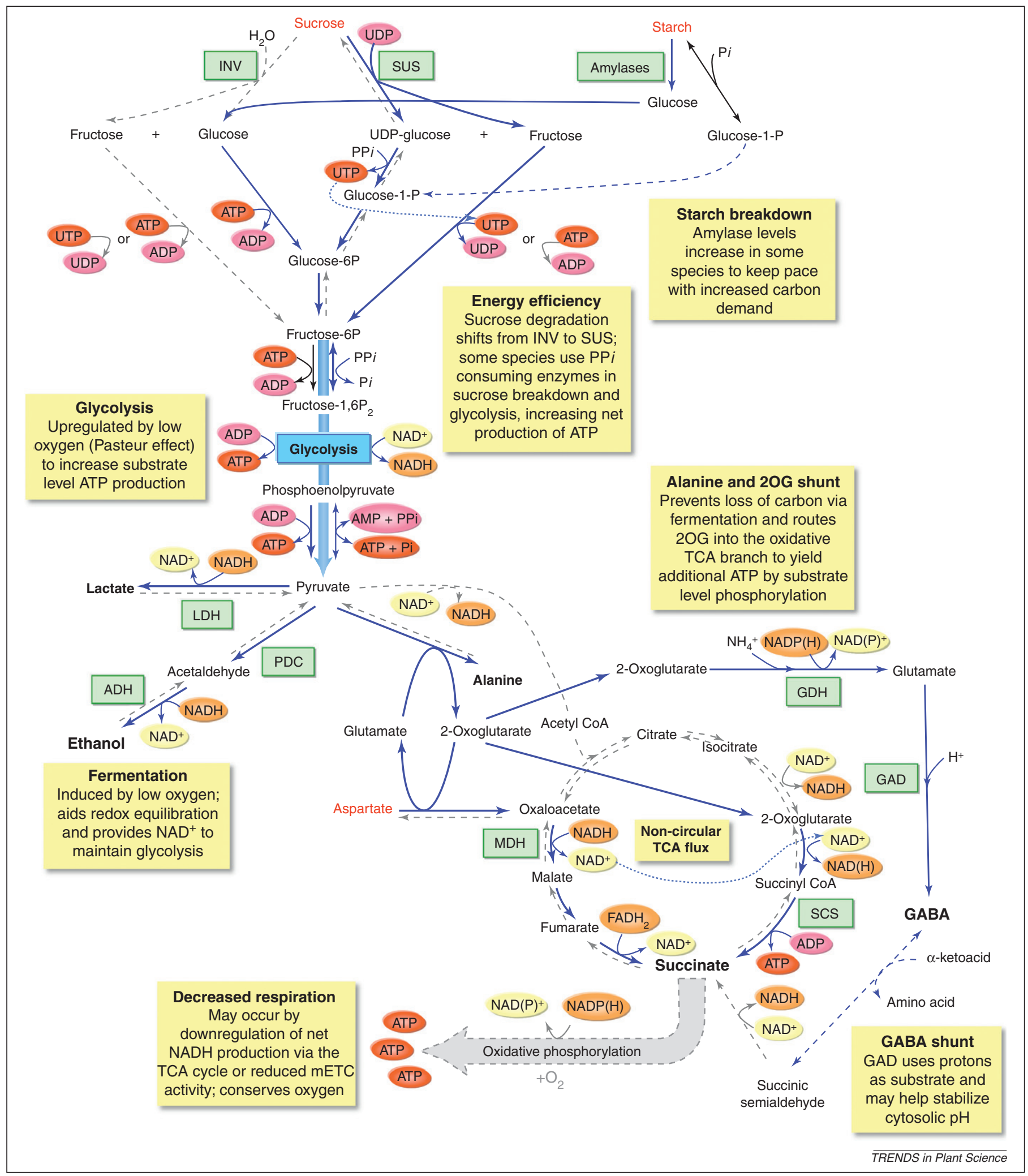

Figure 2. Metabolic reconfiguration under low oxygen stress. Reduced oxygen availability alters metabolism to maximize substrate level ATP production. The model depicts the major known changes that include enhanced sucrose-starch metabolism, glycolysis, fermentation, a modified tricarboxylic acid (TCA) flow, an alanine and 2oxoglutarate (2OG) shunt and a $\gamma$-aminobutyric acid (GABA) shunt. The hypothesis that oxygen is conserved is under further investigation. Yellow boxes summarize notable metabolic adjustments. Blue lines indicate pathways enhanced during the stress, blue dashed lines indicate pathways proposed to be active during the stress and gray dashed lines indicate reactions that are inhibited during the stress. Metabolites that increase during the stress are shown in enlarged black font; metabolites that decrease are shown in red font. Abbreviations are as follows: 2OG, 2-oxoglutarate; ADH, alcohol dehydrogenase; GAD, glutamic acid decarboxylase; GDH, glutamate dehydrogenase, INV, invertase; LDH, lactate dehydrogenase; MDH, malate dehydrogenase; PDC, pyruvate decarboxylase; SCS, succinyl CoA ligase; SUS, sucrose synthase. 
acid (ABA) and gibberellins (GA), which normally limits cell elongation. In rice, natural variation in the presence and absence of the underwater escape [SNORKEL (SK) 1 and 2] and submergence tolerance (SUB1A) group VII ERF determinants underlies differential regulation of the hormonal cascade and hormone sensitivities that control underwater growth [8] (Box 1). Two R. palustris populations distinguished by fast and slow underwater petiole elongation were differentiated by the maintenance of higher levels of ABA and reduced GA responsiveness in the slow elongating variety during submergence [38]. Consistently, the limited underwater petiole growth and prolonged submergence survival of $R$. acetosa was linked to maintenance of ABA biosynthesis that translated into lower GA responsiveness during submergence [36].

Photosynthesis can continue in submerged leaves and is aided by the gas film that often clings to their surface $[39,40]$. It follows that the degree of oxygen deprivation in photosynthetic tissue may be less extreme than tissues distant from an oxygen source. Nevertheless, the ethylenedriven underwater elongation of shoot tissue can deplete carbohydrates and lead to an energy crisis.

\section{Similarities in transcriptome response to flooding and oxygen deprivation}

Numerous investigations have assessed changes in transcriptomes in response to low oxygen stress or flooding in plants including Arabidopsis, rice, poplar (Populus $\times$ canescens) and cotton (Gossypium hirsutum) [41,42]. Studies performed on seedlings of the Arabidopsis Col-0 ecotype include evaluation of the effects of different severity [43] and duration [28,44] of oxygen depletion, as well as the impact of heat stress prior to anoxia [29]. Because the majority of cellular mRNAs are poorly translated during oxygen deprivation [45], changes in polyribosome-associated mRNAs were used to evaluate dynamics in the stress and recovery responses [28]. In 21 cell types or regions of roots and shoots, polyribosomes were captured by immunopurification to identify transcripts regulated by shortterm oxygen deprivation [46]. This approach identified 49 core hypoxia-responsive genes that were strongly induced by the stress across all samples evaluated. Also distinguished were cohorts of mRNAs that were hypoxia-responsive at the organ or cell-specific level, although their modulation was less pronounced than the core

\section{Box 1. Contrasting submergence survival strategies of rice}

Most accessions respond to submergence through rapid shoot elongation, which allows emergence from a shallow flood [6]. A limited number of accessions display the ability to survive a slow progressive flood (escape response) or a deep transient flash flood (quiescence response) (Figure I). (a) By amplifying the elongation of stem internodes, deepwater rice can outgrow a progressive flood and survive partial inundation for months. This deepwater escape strategy is controlled by the SNORKEL (SK) locus, which encodes two group VII ERFs, SK1 and SK2 [34]. SKs are absent from lowland varieties. (b) The molecular genetic analysis of the submergence-tolerant accession FR13A revealed that the SUBMERGENCE 1 (SUB1) locus, encoding two or three group VII ERFs, regulates the quiescence response. SUB1B and $S U B 1 C$ are invariably encoded at $S U B 1$ in lowland accessions, whereas SUB1A is limited to some indica and aus landraces $[2,60]$. The $S U B 1 A-1$ allele is sufficient to confer survival of 2 weeks or longer of complete submergence. (c) Model of the core submergence response network that is influenced by $S K s$ and SUB1A. Genotypes possess either $S K 1 / S K 2, S U B 1 A$ or neither. Both $S K 1 / S K 2$ and SUB1A-1 mRNA are ethylene induced. In deepwater rice, $S K 1 /$ $S K 2$ and two minor QTLs augment accumulation of bioactive GA in stem internodes during submergence. In submergence tolerant rice varieties, the presence of SUB1A-1 influences submergence and postsubmergence responses in aerial tissue. (1) SUB1A-1 mRNA is ethylene-induced but ultimately limits ethylene biosynthesis [37]. (2) SUB1A-1 promotes accumulation of two negative regulators of GA responses [SLENDER RICE 1 (SLR1) and SLENDER RICE-LIKE 1 (SLRL1)] [73]. (3) SUB1A-1 does not perturb the submergenceinduced decline in $A B A$ content but heightens sensitivity to $A B A$ [32]. (4) SUB1A-1 limits induction of genes associated with starch breakdown $[37,41,94]$. (5) SUB1A-1 enhances upregulation of genes associated with reactive oxygen species (ROS) amelioration and survival of dehydration, thereby improving re-establishment following de-submergence [32]. (6) SUB1A-1 interacts with a complex network of proteins [95]. (7) $S U B 1 A-1$ transiently restricts the progression to flowering during submergence [96]. In summary, SUB1A is remarkably positioned to suspend growth and maintain cell viability during submergence and restore homeostasis during a subsequent recovery period. (a)

$$
\text { Progressive flood - deepwater rice (SK1/2) }
$$

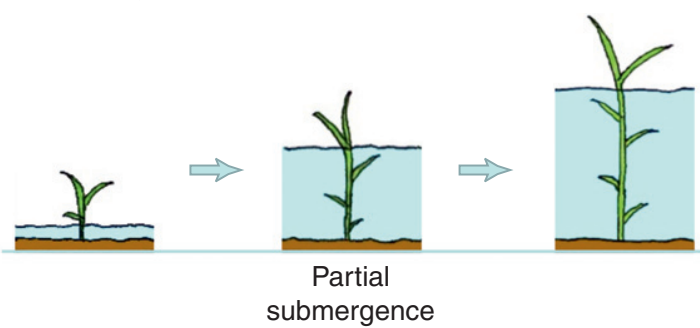

(b)

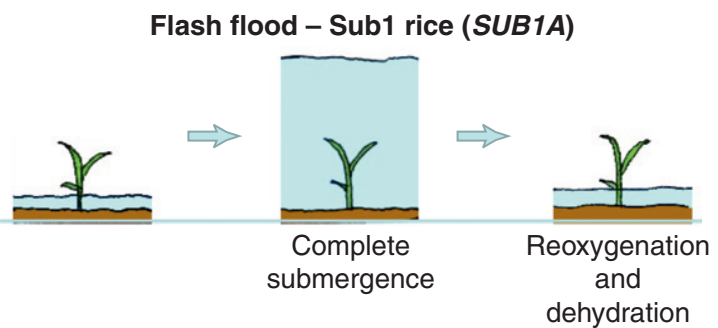

(c)
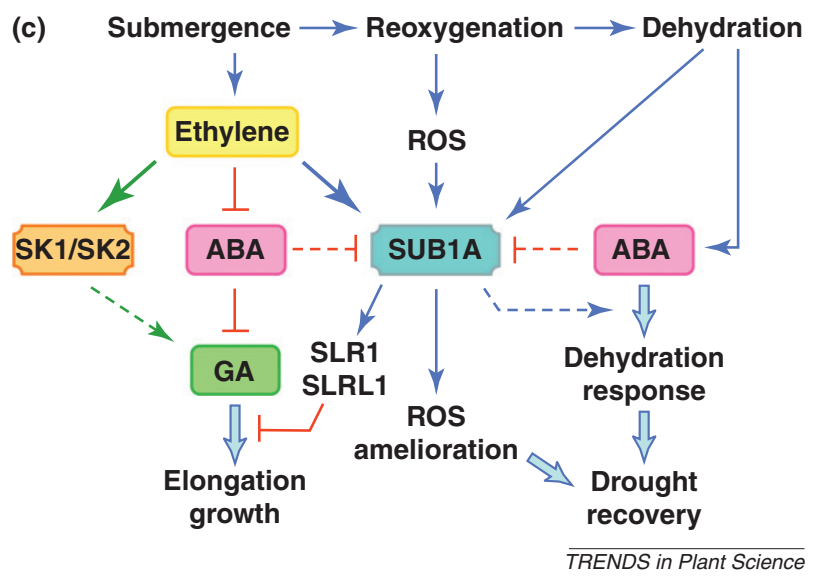

Figure I. Group VII ERFs and pathways that regulate growth responses under distinct flooding regimes. 
hypoxia-responsive genes. Of the 49 core hypoxia-responsive genes, 24 were also differentially regulated in roots and rosette leaves during submergence in complete darkness [47]. Finally, meta-analyses that compared transcriptomic adjustments to low oxygen or flooding stress identified conservation in the core network of genes associated with signaling, transcription and efficient anaerobic ATP production that is modulated by oxygen deprivation in a range of plants $[41,42]$.

\section{How do plant cells sense low oxygen stress?}

Based on mechanisms in other eukaryotes, both indirect and direct sensing of cellular oxygen status could be responsible for acclimation responses that prolong survival of oxygen deprivation in plants [48]. Indirect sensing mechanisms might include perception of altered energy status through changes in levels of adenylates (ATP, ADP and/or AMP), consumable carbohydrates, pyruvate, cytosolic $\mathrm{pH}$, cytosolic $\mathrm{Ca}^{2+}$ or localized production of reactive oxygen species (ROS) and nitric oxide (NO).

Animal and yeast cells sense and adjust energy homeostasis through Sucrose Non-Fermenting 1 (SNF1)/AMPactivated protein kinases $[49,50]$. The plant energy sensors fall within one clade of SNF1 relatives, the SnRK1s, some of which have been implicated in low oxygen responses. For example, Arabidopsis KIN10 and KIN11 are necessary to limit energy consumption during hypoxia [51]. Whereas in rice seeds germinated under oxygen starvation, the depletion of sucrose activates the SnRK1A energy sensor through the activity of a Calcineurin B-like interacting binding kinase 15 (CIPK15) [52]. This signal transduction upregulates transcription of genes encoding $\alpha$-amylases, which drive catabolism of starch in the seed needed to fuel underwater shoot growth. Logically, a reduction of energy consumption is beneficial when ATP levels decline. A means of energy conservation during low oxygen stress in plants is selective translation and sequestration of mRNAs during hypoxia [28]. Based on evidence from other eukaryotes, the sequestration of a subset of cellular mRNAs, such as the abundant cohort that encodes ribosomal proteins and translation factors, could be regulated through SnRK1s and the Target of Rapamycin kinase [53]. Mitochondria are also thought to contribute to oxygen sensing and signaling in plants, through production of $\mathrm{NO}$ and/or release of ROS and $\mathrm{Ca}^{2+}$ during the transition from normoxia to hypoxia $[54,55]$, as confirmed in animals $[56,57]$.

In animals, direct oxygen sensing regulates the accumulation of the $\alpha$ subunit of the hypoxia inducible factor (HIF) $1 \alpha / \beta$ transcription factor [58]. HIF $1 \alpha$ is constitutively synthesized but fails to accumulate under normoxia because of oxygen-dependent hydroxylation of specific proline residues that trigger its ubiquitination and $26 \mathrm{~S}$ proteasome-mediated degradation. As oxygen declines, the prolyl hydroxylases that modify HIF $1 \alpha$ are less active. Consequentially, HIF $1 \alpha$ accumulates and is trafficked to the nucleus where HIF $1 \alpha / \beta$ can function in transcriptional activation. There is no corollary direct oxygen sensing mechanism in plants, because although they possess prolyl hydroxylases they lack HIF $1 \alpha[41]$.

\section{Group VII ERFs regulate low-oxygen acclimation responses}

The plant-specific ERF transcription factor family includes over 100 members in rice and Arabidopsis, all of which share an APETALA2 (AP2) DNA binding domain [59]. The ERFs have been phylogenetically parsed into ten clades, with the group VII ERFs characterized by a conserved $\mathrm{N}$ terminal motif $\left(\mathrm{NH}_{2}-\mathrm{MCGGAI} / \mathrm{L}\right)$ [59] (Figure 3a). Fifteen rice (japonica cv. Nipponbare) ERFs were designated group VIIa (OsERF59-72) and VIIb (OsERF73), based on the presence or absence of the conserved $\mathrm{N}$ terminal motif, respectively. The single group VIIb ERF corresponds to $S U B 1 C$, which is found in all rice varieties surveyed [60] and acts downstream of SUB1A [37]. Intriguingly, the group VII ERFs encoded by $S U B 1 A, S K 1$ and $S K 2$ possess variant $\mathrm{N}$ termini relative to the rice group VIIa ERFs. Arabidopsis encodes five group VII ERFs (AtERF71-75), two of which are hypoxia-responsive genes [HYPOXIA RESPONSIVE ERF1 and 2 [HRE1 (AtERF73; At1g72360) and HRE2 (AtERF71; At2g47520)]\}. As observed for SUB1A and the SKs, HRE1 mRNA accumulation is promoted by ethylene, which synergistically enhances its elevation during hypoxia [61,62] (Figure 3b).

Several recent reports indicate that Arabidopsis group VII ERFs redundantly regulate hypoxia-responsive gene expression and survival of low oxygen stress. For example, seedling survival of anoxia was more severely compromised in hre1hre2 double mutant seedlings than in either single mutant or the wild type $[61,63]$. By contrast, low oxygen sensitivity was lessened in seedlings that constitutively overexpress either HRE1 or HRE2 mRNA. The ectopic expression of these ERFs was sufficient to heighten induction of the core hypoxia-responsive gene $A D H 1$ or $\mathrm{ADH}$ enzyme activity during the stress $[61,63]$. However, because hre1hre 2 seedlings were able to elevate $\mathrm{ADH}$ enzyme activity and ethanol production during hypoxia [63], genetic redundancy is likely to extend to the other group VII ERFs [RAP2.12 (AtERF75; At1g53910), RAP2.2 (AtERF74; At3g14230) and RAP2.3 (AtEBP/AtERF72; At3g16770)]. Indeed, conditional upregulation of $R A P 2.12$ was sufficient to elevate expression of a $p A D H 1$ :LUCIFERASE transgene [64] and RAP2.2 overexpression improved survival of hypoxia in seedlings [65], whereas the inhibition of either RAP2.2 or RAP2.12 expression via miRNA production limited the induction of $A D H 1$ and several other hypoxia-responsive genes [66]. The impact of ectopic expression of these genes was condition specific, as HRE1, HRE2, RAP2.2 and RAP2.12 overexpression significantly increased levels of $A D H 1$ mRNA or ADH activity under low oxygen stress but not under normoxia [61,65,66]. Nonetheless, RAP2.2, RAP2.3 and RAP2.12 mRNAs accumulate under normoxia in association with polyribosomes [46,67], suggesting they are constitutively synthesized. Together, these findings hint that post-translational regulation limits the function of group VII ERFs to periods of low oxygen stress.

\section{Arabidopsis group VII ERFs are degraded via the $\mathrm{N}$-end rule pathway}

The conserved N-end rule pathway of targeted proteolysis regulates the half-life of certain cellular proteins based on 


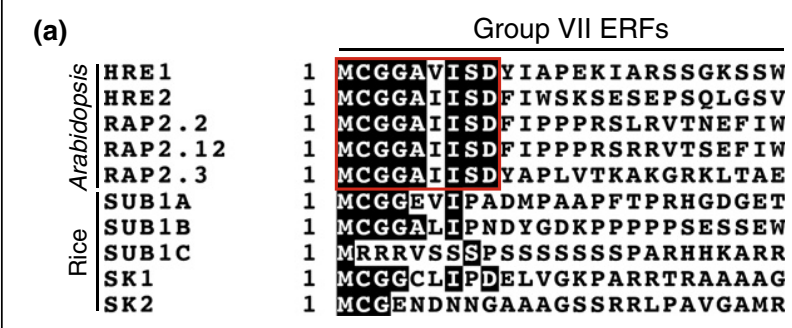

(b)
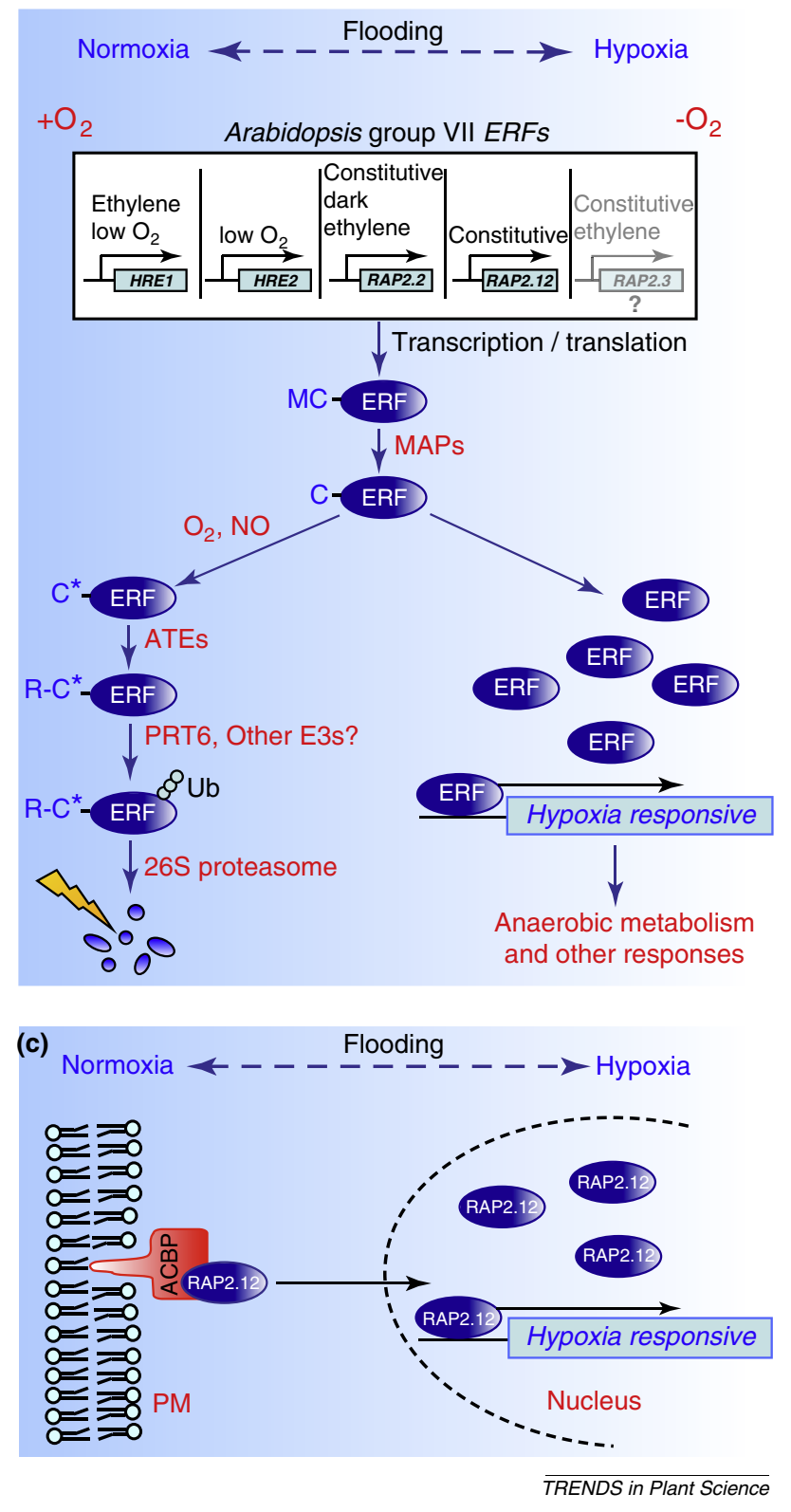

Figure 3. Oxygen sensing via $\mathrm{N}$-end rule pathway-targeted turnover of group VII ERFs. (a) $\mathrm{N}$ terminal alignment of Arabidopsis group VII ERFs. With the exception of SUB1C, all begin with the amino acids 'Met-Cys' (MC). The highly conserved Arabidopsis $\mathrm{N}$ terminal motif is boxed in red and is less conserved in the proteins at loci associated with submergence responses in rice. (b) Homeostatic response to hypoxia is regulated by the N-end rule-mediated proteolysis of group VII ERFs in Arabidopsis. Group VII ERF transcription factors are either constitutively expressed and/or differentially transcriptionally regulated in response to variable signals, including low $\mathrm{O}_{2}$, ethylene and darkness. Four of the five ERFs (HRE1, HRE2, RAP2.2 and RAP2.12) have been implicated in the regulation of hypoxia-responsive genes. Under oxygen-replete conditions (normoxia), ERFs are degraded via the Nend rule pathway of proteolysis. This involves the following steps: (i) the $N$ terminal Met $(\mathrm{M})$ is constitutively cleaved by a methionine aminopeptidase (MAP) (ii) the exposed Cys (C) is converted to an oxidized $\left(C^{*}\right)$ form (e.g. Cys-sulfonic acid) by $\mathrm{O}_{2}$, NO or possibly $\mathrm{ROS}$; (iii) an $\operatorname{Arg}(\mathrm{R})$ residue is added to the oxidized recognition of $\mathrm{N}$ terminal residues by specific $\mathrm{N}$-recognin E3 ligases [68]. In plants, 11 amino acids function as destabilizing residues when located at the $\mathrm{N}$ terminus of a protein, which coupled with an optimally positioned downstream lysine can act as a degradation signal (Ndegron) [69]. In plants and animals but not yeast, a cysteine (Cys) residue at the $\mathrm{N}$ terminus can undergo two steps of modification that lead to protein recognition and degradation (Figure 3b). Based on mechanistic studies in mammals, newly synthesized proteins with a Cys as the second residue (i.e. $\mathrm{NH}_{2}-\mathrm{Met}_{1}-\mathrm{Cys}_{2}$ ) are constitutively cleaved by a Met amino peptidase (MAP) to yield $\mathrm{NH}_{2}-\mathrm{Cys}_{2}$. In Arabidopsis, a small family of functionally redundant MAPs catalyzes this reaction [70]. The exposed $\mathrm{Cys}_{2}$ can be spontaneously or enzymatically oxidized in an $\mathrm{O}_{2^{-}}$or NO-dependent manner to Cys-sulfinate or further to Cys-sulfonate [68]. As a result of oxidation, an arginine residue is added to the $\mathrm{NH}_{2}-\mathrm{Cys}_{2}$ by an arginyl tRNA transferase (ATE), targeting the protein for recognition by an N-recognin E3 ligase, leading to ubiquitination and 26S proteasome-mediated degradation. In Arabidopsis, the genes ATE1 and ATE2 encode the Arg transferases [71] and at least one E3 ligase, encoded by PROTEOLYSIS 6 (PRT6), acts as an N-recognin of $\mathrm{NH}_{2}-\mathrm{Arg}_{1}-\mathrm{Cys}_{2}{ }^{\text {oxidised }}$ polypeptides [69].

The distinct conservation of the $\mathrm{N}$ terminus of group VII ERFs and the serendipitous observation that an Arabidopsis prt6 mutant constitutively accumulates $A D H 1$ and other hypoxia-responsive mRNAs in seeds led to the confirmation that group VII ERFs are bona fide substrates of the N-end rule pathway in plants [66,72] (Figure 3b). Additional support of this conclusion was obtained through in vitro and in planta analyses.

An in vitro system derived from rabbit reticulocytes [72] was used to confirm that all five Arabidopsis group VII ERFs are N-end rule substrates. It was also shown that their instability required $\mathrm{Cys}_{2}$, as mutation of $\mathrm{Cys}_{2}$ to the stabilizing residue $\mathrm{Ala}_{2}\left(\mathrm{NH}_{2}-\mathrm{Met}_{1}-\mathrm{Ala}_{2}\right)$ eliminated susceptibility to $\mathrm{N}$-end rule turnover. It was further demonstrated in planta that low oxygen stress increased the accumulation of group VII ERFs synthesized with a native $\mathrm{N}$-terminus $\left(\mathrm{NH}_{2}-\mathrm{Met}_{1}-\mathrm{Cys}_{2}\right)$, whereas those synthesized with an $\mathrm{NH}_{2}-\mathrm{Met}_{1}-\mathrm{Ala}_{2} \mathrm{~N}$ terminus were stable under both normoxia and hypoxia [66,72]. Based on this evidence, the stabilization of group VII ERFs under hypoxia is most probably related to an inhibition of the $\mathrm{Cys}_{2}$ oxidation that is required before the protein can be arginylated and degraded.

Cys by an arginyl tRNA transferase (ATE); and (iv) the argininylated protein is recognized by PROTEOLYSIS 6 (PRT6) or other E3 ligases, which polyubiquitinate the protein, targeting it for proteasomal degradation (26S proteasome). The outcome is prevention of transcription of hypoxia-responsive genes under normoxia. When oxygen becomes limiting (hypoxia), degradation of the ERFs by the $\mathrm{N}$-end rule pathway is inhibited due to a lack of oxygen-mediated $\mathrm{Cys}_{2}$ oxidation. Stabilized ERFs can then drive the transcription of genes that enhance anaerobic metabolism and other survival responses. Upon return to aerobic conditions, the ERFs are once again destabilized, providing a feedback mechanism that allows the plant to return to aerobic metabolism. (c) AtRAP2.12 localization dynamics. At least one group VII ERF, RAP2.12, associates with the plasma membrane (PM) via interaction with $A C B P$, limiting its turnover under normoxia. During hypoxia RAP2.12 is relocated to the nucleus and activates gene expression. Upon reoxygenation, RAP2.12 is destabilized, presumably as a consequence of $\mathrm{Cys}_{2}$ oxidation and $\mathrm{N}$-end rule-mediated degradation. 
Either the modification of the $\mathrm{N}$ terminus of a group VII $\mathrm{ERF}$ or disruption of an $\mathrm{N}$-end rule pathway step can affect survival of low oxygen stress or submergence in Arabidopsis [66]. For example, stabilization of HRE1 and HRE2 by modification of the $\mathrm{N}$ terminus to $\mathrm{NH}_{2}-\mathrm{Met}_{1}-\mathrm{Ala}_{2}$ was sufficient to improve seed germination and seedling survival under hypoxia [72]. In addition, ate1ate2 and prt6 seedlings were less sensitive to hypoxia when grown on sucrose-supplemented medium [72]. The same mutants grown to the rosette stage were more sensitive to submergence in complete darkness [66]. This discrepancy in phenotype might be explained by distinctions in the available carbohydrates in the two survival assays. In the low oxygen experiments, anaerobic metabolism was fueled by sucrose in the medium, whereas in the submergence experiments it was limited to endogenous energy reserves of the plant. Therefore, the absence of PRT6 or ATE activity may enhance anaerobic metabolism to prolong survival in sucrose-fed seedlings but may cause a more rapid onset of energy deficiency in submerged plants. These findings are reminiscent of the earlier proposal that a balance between energy consumption and conservation is crucial to survival of low oxygen stress and submergence $[5,37]$.

It was also observed that the onset of the transcription of hypoxia-responsive genes occurs concomitantly with relocalization of RAP2.12 to the nucleus under hypoxia (Figure 3c) [66]. During normoxia, a GFP-tagged version of RAP2.12 was protected against protein degradation by the $\mathrm{N}$-end rule pathway of proteolysis and excluded from the nucleus via interaction with a plasma membrane (PM)associated Acyl-CoA binding protein (ACBP1 or ACBP2). RAP2.12 migrated to the nucleus in response to hypoxia and disappeared from the nucleus after reoxygenation. Moreover, transient expression of RAP2.12-GFP in leaves of ate1ate2 and prt6 mutants resulted in greater GFP signal intensity in the nucleus under normoxia and following reoxygenation.

In summary, the $\mathrm{N}$-end rule pathway of proteolysis regulates the accumulation of group VII ERFs and consequentially the accumulation of gene transcripts associated with low oxygen responses in Arabidopsis. It is proposed that constitutively synthesized group VII ERFs are either degraded or sequestered under normoxia, as confirmed for $R A P 2.12$. As oxygen levels fall their degradation becomes limited, PM sequestration is reversed and the ERF is transported to the nucleus and becomes active in gene regulation. Upon reoxygenation, both constitutively expressed and hypoxia-induced group VII ERFs are destabilized. Thus, the N-end rule pathway (i) prevents the excessive accumulation of constitutively expressed ERFs under normoxia; (ii) allows for stabilization of both constitutive and induced ERFs during hypoxia; and (iii) facilitates rapid reversal of ERF-regulated transcription upon reoxygenation. Constitutively expressed group VII ERFs are proposed to encode oxygen sensors that conditionally activate transcription of hypoxia-responsive genes, including other group VII ERFs [66]. The increased synthesis of $\mathrm{N}$-end rule regulated group VII ERFs by ethylene or darkness could further prime cells for acclimation to oxygen deprivation.

\section{Manipulation of $\mathrm{N}$-end rule regulation of group VII ERFs and other proteins}

The verification that the $\mathrm{N}$-end rule pathway modulates group VII ERF accumulation in the nucleus in an oxygendependent manner exposes the first examples of $\mathrm{N}$-end rule substrates and a homeostatic low oxygen sensor mechanism in plants. Based on available gene sequence data, group VII ERFs with the conserved N-terminus are broadly found in vascular plant species [66]. We propose that future improvement of flooding tolerance could be achieved by manipulation of synthesis and turnover of these proteins (e.g. by overexpression, regulated expression and/or mutation of $\mathrm{NH}_{2}-\mathrm{Met}_{1}-\mathrm{Cys}_{2}$ to $\mathrm{NH}_{2}-\mathrm{Met}_{1}-\mathrm{Ala}_{2}$ ).

Given the crucial importance of modulation of energy reserves during flooding, it is not surprising that variation of group VII ERF susceptibility to oxygen-dependent Nend rule turnover exists in nature. The rice Nipponbare genome encodes 15 group VII ERFs with the conserved N terminus that is consistent with oxygen-regulated $\mathrm{N}$-end rule-targeted proteolysis in Arabidopsis. However, neither SUB1A nor SUB1C are N-end rule substrates based on in vitro data [72] and the $\mathrm{N}$ termini of SK1 and SK2 also deviate from the consensus associated with $\mathrm{N}$-end rulemediated turnover. This leads us to propose that the escape of SUB1A from N-end rule pathway turnover could allow the ethylene-mediated regulation of SUB1A-1 to trigger the sequence of events that promotes the energy management associated with submergence tolerance well before oxygen levels reach a critical nadir.

\section{Concluding remarks: direct oxygen sensing via the $\mathbf{N}$ - end rule regulates transcription}

Alterations in gene expression associated with increased catabolism and substrate level ATP production are a hallmark of reduced oxygen availability and flooding in plants. Group VII ERFs play a prominent role in this process. The identification of an oxygen-dependent protein turnover mechanism that controls the abundance of some but not all group VII ERFs raises several pertinent questions (Box 2). We anticipate that genetic manipulation of the targets of oxygen-regulated $\mathrm{N}$-end rule pathway turnover can provide a means to improve survival under a variety of flooding conditions.

\section{Box 2. Key questions for future experimentation}

- Can $\mathrm{O}_{2}, \mathrm{NO}$ or ROS-dependent $\mathrm{Cys}_{2}$ oxidation, arginylation and ubiquitination of group VII ERFs be experimentally confirmed? If so, is the oxidation spontaneous or catalyzed?

- What are the kinetics of oxygen-regulated group VII ERF turnover? Does ERF stabilization occur before oxygen deficiency impairs cytochrome $c$ oxidase activity?

- Does the oxygen level affect the interaction between ACBPs and RAP2.12? Does docking of RAP2.12 to ACBP impair $\mathrm{Cys}_{2}$ oxidation, modification by ATE, or interaction with an E3 ligase? Are other group VII ERFs similarly sequestered?

- What genes and networks are controlled by individual group VII ERFs?

- Is the activity or turnover of SUB1A, which apparently escapes oxygen-mediated $\mathrm{N}$-end rule degradation, controlled upon desubmergence?

- Can manipulation of group VII ERF accumulation and turnover provide an effective strategy to modulate survival of flooding in crops? 


\section{Acknowledgments}

Submergence and hypoxia research in the Bailey-Serres group is supported by the National Science Foundation (IOS-0750811; IOS1121626) and the United States Department of Agriculture (200835100-04528), in the van Dongen group by the German Research Foundation (DFG; DO 1298/2-1) and the Federal Ministry of Education and Research (BMBF; HydromicPro), in the Perata group by Scuola Superiore Sant'Anna (A6010PP-A6011PP), and in the Voesenek group by grants from the Netherlands Organization for Scientific Research and Center for BioSystems and Genomics (CBSG2012). N-end rule pathway research in the Holdsworth group is supported by the Biotechnology and Biological Sciences Research Council (BB/G010595/1).

\section{References}

1 Tester, M. and Langridge, P. (2010) Breeding technologies to increase crop production in a changing world. Science $327,818-822$

$2 \mathrm{Xu}$, K. et al. (2006) Sub1A is an ethylene-response-factor-like gene that confers submergence tolerance to rice. Nature 442, 705-708

3 Septiningsih, E.M. et al. (2009) Development of submergence-tolerant rice cultivars: the Sub1 locus and beyond. Ann. Bot. 103, 151-160

4 Bailey-Serres, J. et al. (2010) Submergence tolerant rice: SUB1's journey from landrace to modern cultivar. Rice 3, 138-147

5 Fukao, T. and Bailey-Serres, J. (2004) Plant responses to hypoxia - is survival a balancing act? Trends Plant Sci. 9, 449-456

6 Bailey-Serres, J. and Voesenek, L.A.C.J. (2008) Flooding stress: acclimations and genetic diversity. Annu. Rev. Plant Biol. 59, 313-339

7 Colmer, T.D. and Voesenek, L.A.C.J. (2009) Flooding tolerance: suites of plant traits in variable environments. Funct. Plant Biol. 36, 665-681

8 Bailey-Serres, J. and Voesenek, L.A.C.J. (2010) Life in the balance: a signaling network controlling survival of flooding. Curr. Opin. Plant Biol. 13, 489-494

9 Colmer, T.D. (2003) Long-distance transport of gases in plants: a perspective on internal aeration and radial oxygen loss from roots. Plant Cell Environ. 26, 17-36

10 Gupta, K.J. et al. (2009) Regulation of respiration when the oxygen availability changes. Physiol. Plant. 137, 383-391

11 Malik, A.I. et al. (2011) Transfer of the barrier to radial oxygen loss in roots of Hordeum marinum to wheat (Triticum aestivum): evaluation of four H. marinum-wheat amphiploids. New Phytol. 190, 499-508

12 Zabalza, A. et al. (2009) Regulation of respiration and fermentation to control the plant internal oxygen concentration. Plant Physiol. 149, 1087-1098

13 Geigenberger, P. (2003) Response of plant metabolism to too little oxygen. Curr. Opin. Plant Biol. 6, 247-256

14 van Dongen, J.T. et al. (2003) Phloem metabolism and function have to cope with low internal oxygen. Plant Physiol. 131, 1529-1543

15 van Dongen, J.T. et al. (2004) Phloem import and storage metabolism are highly coordinated by the low oxygen concentrations within developing wheat seeds. Plant Physiol. 135, 1809-1821

16 Magneschi, L. and Perata, P. (2009) Rice germination and seedling growth in the absence of oxygen. Ann. Bot. 103, 181-196

17 Ricoult, C. et al. (2006) Characterization of alanine aminotransferase (AlaAT) multigene family and hypoxic response in young seedlings of the model legume Medicago truncatula. J. Exp. Bot. 57, 3079-3089

18 Limami, A.M. et al. (2008) Concerted modulation of alanine and glutamate metabolism in young Medicago truncatula seedlings under hypoxic stress. J. Exp. Bot. 59, 2325-2335

19 Rocha, M. et al. (2010) Glycolysis and the tricarboxylic acid cycle are linked by alanine aminotransferase during hypoxia induced by waterlogging of Lotus japonicus. Plant Physiol. 152, 1501-1513

20 Sweetlove, L.J. et al. (2010) Not just a circle: flux modes in the plant TCA cycle. Trends Plant Sci. 15, 462-470

21 Vanlerberghe, G.C. et al. (1989) Anaerobic carbon metabolism by the tricarboxylic acid cycle: evidence for partial oxidative and reductive pathways during dark ammonium assimilation. Plant Physiol. 91, $1551-1557$

22 Ramirez-Aguilar, S.J. et al. (2011) The composition of plant mitochondrial supercomplexes changes with the oxygen availability. J. Biol. Chem. 286, 43045-43053

23 Felle, H.H. (2006) Apoplastic pH during low-oxygen stress in Barley. Ann. Bot. 98, 1085-1093

24 Kulichikhin, K.Y.et al. (2008) Intracellular pH in rice and wheat root tips under hypoxic and anoxic conditions. Plant Signal. Behav. 3, 240-242
25 Guglielminetti, L. et al. (1995) Effect of anoxia on carbohydrate metabolism in rice seedlings. Plant Physiol. 108, 735-741

26 Huang, S. et al. (2008) Does anoxia tolerance involve altering the energy currency towards PPi? Trends Plant Sci. 13, 221-227

27 Rolletschek, H. et al. (2011) Combined noninvasive imaging and modeling approaches reveal metabolic compartmentation in the barley endosperm. Plant Cell 23, 3041-3054

28 Branco-Price, C. et al. (2008) Selective mRNA translation coordinates energetic and metabolic adjustments to cellular oxygen deprivation and reoxygenation in Arabidopsis thaliana. Plant J. 56, 743-755

29 Banti, V. et al. (2010) The heat-inducible transcription factor HsfA2 enhances anoxia tolerance in Arabidopsis. Plant Physiol. 152, 14711483

30 Gajdanowicz, P. et al. (2011) Potassium (K+) gradients serve as a mobile energy source in plant vascular tissues. Proc. Natl. Acad. Sci. U.S.A. 108, 864-869

31 Blokhina, O. et al. (2003) Antioxidants, oxidative damage and oxygen deprivation stress: a review. Ann. Bot. 91, 179-194

32 Fukao, T. et al. (2011) The submergence tolerance regulator SUB1A mediates crosstalk between submergence and drought tolerance in rice. Plant Cell 23, 412-427

33 Licausi, F. (2011) Regulation of the molecular response to oxygen limitations in plants. New Phytol. 190, 550-555

34 Hattori, Y. et al. (2009) The ethylene response factors SNORKEL1 and SNORKEL2 allow rice to adapt to deep water. Nature 460, 1026-1030

35 Nagai, K. et al. (2010) Stunt or elongate? Two opposite strategies by which rice adapts to floods. J. Plant Res. 123, 303-309

36 Benschop, J.J. et al. (2005) Contrasting interactions between ethylene and abscisic acid in Rumex species differing in submergence tolerance. Plant J. 44, 756-768

37 Fukao, T. et al. (2006) A variable cluster of ethylene response factor-like genes regulates metabolic and developmental acclimation responses to submergence in rice. Plant Cell 18, 2021-2034

38 Chen, X. et al. (2010) Endogenous abscisic acid as a key switch for natural variation in flooding-induced shoot elongation. Plant Physiol. 154, 969-977

39 Pedersen, O. et al. (2009) Surviving floods: leaf gas films improve $\mathrm{O}(2)$ and $\mathrm{CO}(2)$ exchange, root aeration, and growth of completely submerged rice. Plant J. 58, 147-156

40 Winkel, A. et al. (2011) Leaf gas films of Spartina anglica enhance rhizome and root oxygen during tidal submergence. Plant Cell Environ. $34,83-92$

41 Mustroph, A. et al. (2010) Cross-kingdom comparison of transcriptomic adjustments to low-oxygen stress highlights conserved and plantspecific responses. Plant Physiol. 152, 1484-1500

42 Narsai, R. et al. (2011) Comparative analysis between plant species of transcriptional and metabolic responses to hypoxia. New Phytol. 190, $472-487$

43 van Dongen, J.T. et al. (2009) Transcript and metabolite profiling of the adaptive response to mild decreases in oxygen concentration in the roots of Arabidopsis plants. Ann. Bot. 103, 269-280

$44 \mathrm{Liu}, \mathrm{F}$. et al. (2005) Global transcription profiling reveals comprehensive insights into hypoxic response in Arabidopsis. Plant Physiol. 137, 1115-1129

45 Branco-Price, C. et al. (2005) Genome-wide analysis of transcript abundance and translation in Arabidopsis seedlings subjected to oxygen deprivation. Ann. Bot. 96, 647-660

46 Mustroph, A. et al. (2009) Profiling translatomes of discrete cell populations resolves altered cellular priorities during hypoxia in Arabidopsis. Proc. Natl. Acad. Sci. U.S.A. 106, 18843-18848

47 Lee, S.C. et al. (2011) Molecular characterization of the submergence response of the Arabidopsis thaliana ecotype Columbia. New Phytol. $190,457-471$

48 Bailey-Serres, J. and Chang, R. (2005) Sensing and signalling in response to oxygen deprivation in plants and other organisms. Ann. Bot. 96, 507-518

49 Carling, D. et al. (2011) AMP-activated protein kinase: nature's energy sensor. Nat. Chem. Biol. 7, 512-518

50 Hardie, D.G. (2011) Cell biology. Why starving cells eat themselves. Science 331, 410-411

51 Baena-Gonzalez, E. et al. (2007) A central integrator of transcription networks in plant stress and energy signalling. Nature 448, 938942 
52 Lee, K.W. et al. (2009) Coordinated responses to oxygen and sugar deficiency allow rice seedlings to tolerate flooding. Sci. Signal. 2, ra61

53 Baena-Gonzalez, E. (2010) Energy signaling in the regulation of gene expression during stress. Mol. Plant 3, 300-313

54 Rhoads, D.M. and Subbaiah, C.C. (2007) Mitochondrial retrograde regulation in plants. Mitochondrion 7, 177-194

55 Blokhina, O. and Fagerstedt, K.V. (2010) Oxidative metabolism, ROS and NO under oxygen deprivation. Plant Physiol. Biochem. 48, 359-373

56 Chandel, N.S. (2010) Mitochondrial regulation of oxygen sensing. Adv. Exp. Med. Biol. 661, 339-354

57 Mungai, P.T. et al. (2011) Hypoxia triggers AMPK activation through reactive oxygen species-mediated activation of calcium releaseactivated calcium channels. Mol. Cell. Biol. 31, 3531-3545

58 Kaelin, W.G., Jr and Ratcliffe, P.J. (2008) Oxygen sensing by metazoans: the central role of the HIF hydroxylase pathway. Mol. Cell 30, 393-402

59 Nakano, T. et al. (2006) Genome-wide analysis of the ERF gene family in Arabidopsis and rice. Plant Physiol. 140, 411-432

60 Singh, N. et al. (2010) Molecular marker survey and expression analyses of the rice submergence-tolerance gene SUB1A. Theor. Appl. Genet. 121, 1441-1453

61 Hess, N. et al. (2011) The hypoxia responsive transcription factor genes ERF71/HRE2 and ERF73/HRE1 of Arabidopsis are differentially regulated by ethylene. Physiol. Plant. 143, 41-49

62 Yang, C.Y. et al. (2011) The AP2/ERF transcription factor AtERF73/ HRE1 modulates ethylene responses during hypoxia in Arabidopsis. Plant Physiol. 156, 202-212

63 Licausi, F. et al. (2010) HRE1 and HRE2, two hypoxia-inducible ethylene response factors, affect anaerobic responses in Arabidopsis thaliana. Plant J. 62, 302-315

64 Papdi, C. et al. (2008) Functional identification of Arabidopsis stress regulatory genes using the controlled cDNA overexpression system. Plant Physiol. 147, 528-542

65 Hinz, M. et al. (2010) Arabidopsis RAP2.2: an ethylene response transcription factor that is important for hypoxia survival. Plant Physiol. 153, 757-772

66 Licausi, F. et al. (2011) Oxygen sensing in plants is mediated by an Nend rule pathway for protein destabilisation. Nature 479, 419-422

67 Mustroph, A. and Bailey-Serres, J. (2010) The Arabidopsis translatome cell-specific mRNA atlas: mining suberin and cutin lipid monomer biosynthesis genes as an example for data application. Plant Signal. Behav. 5, 320-324

68 Varshavsky, A. (2011) The N-end rule pathway and regulation by proteolysis. Protein Sci. 20, 298-1345

69 Graciet, E. and Wellmer, F. (2010) The plant N-end rule pathway: structure and functions. Trends Plant Sci. 15, 447-453

70 Ross, S. et al. (2005) Functional and developmental impact of cytosolic protein N-terminal methionine excision in Arabidopsis. Plant Physiol. 137, 623-637

71 Yoshida, S. et al. (2002) A delayed leaf senescence mutant is defective in arginyl-tRNA:protein arginyltransferase, a component of the N-end rule pathway in Arabidopsis. Plant J. 32, 129-137

72 Gibbs, D.J. et al. (2011) Homeostatic response to hypoxia is regulated by the N-end rule pathway in plants. Nature $479,415-418$

73 Fukao, T. and Bailey-Serres, J. (2008) Submergence tolerance conferred by Sub1A is mediated by SLR1 and SLRL1 restriction of gibberellin responses in rice. Proc. Natl. Acad. Sci. U.S.A. 105, 16814-16819

74 Angaji, S.A. et al. (2010) QTLs associated with tolerance of flooding during germination in rice (Oryza sativa L.). Euphytica 172, 159-168

75 Ismail, A.M. et al. (2009) Mechanisms associated with tolerance to flooding during germination and early seedling growth in rice (Oryza sativa). Ann. Bot. 103, 197-209
76 Shingaki-Wells, R.N. et al. (2011) Differential molecular responses of rice and wheat coleoptiles to anoxia reveal novel metabolic adaptations in amino acid metabolism for tissue tolerance. Plant Physiol. 156, 1706-1724

77 Vashisht, D. et al. (2011) Natural variation of submergence tolerance among Arabidopsis thaliana accessions. New Phytol. 190, 299-310

78 Rich, S.M. et al. (2011) Aquatic adventitious roots of the wetland plant Meionectes brownii can photosynthesize: implications for root function during flooding. New Phytol. 190, 311-319

79 Perata, P. et al. (1992) Effect of anoxia on starch breakdown in rice and wheat seeds. Planta 188, 611-618

80 Mus, F. et al. (2007) Anaerobic acclimation in Chlamydomonas reinhardtii: anoxic gene expression, hydrogenase induction, and metabolic pathways. J. Biol. Chem. 282, 25475-25486

81 Dubini, A. et al. (2009) Flexibility in anaerobic metabolism as revealed in a mutant of Chlamydomonas reinhardtii lacking hydrogenase activity. J. Biol. Chem. 284, 7201-7213

82 Chochois, V. et al. (2009) Hydrogen production in Chlamydomonas: photosystem II-dependent and -independent pathways differ in their requirement for starch metabolism. Plant Physiol. 151, 631-640

83 Koizumi, Y. et al. (2011) Involvement of plasma membrane $\mathrm{H}^{+}$-ATPase in anoxic elongation of stems in pondweed (Potamogeton distinctus) turions. New Phytol. 190, 421-430

84 Mugnai, S. et al. (2011) Effect of hypoxic acclimation on anoxia tolerance in Vitis roots: response of metabolic activity and $\mathrm{K}^{+}$fluxes. Plant Cell Physiol. 52, 1107-1116

85 Bond, D.M. et al. (2009) VERNALIZATION INSENSITIVE 3 (VIN3) is required for the response of Arabidopsis thaliana seedlings exposed to low oxygen conditions. Plant J. 59, 576-587

86 Schroder, F. et al. (2011) EXORDIUM-LIKE1 promotes growth during low carbon availability in Arabidopsis. Plant Physiol. 156, 1620-1630

87 Christianson, J.A. et al. (2009) The low-oxygen-induced NAC domain transcription factor ANAC102 affects viability of Arabidopsis seeds following low-oxygen treatment. Plant Physiol. 149, 1724-1738

88 Kreuzwieser, J. et al. (2009) Differential response of gray poplar leaves and roots underpins stress adaptation during hypoxia. Plant Physiol. $149,461-473$

89 Christianson, J.A. et al. (2010) Global gene expression responses to waterlogging in roots and leaves of cotton (Gossypium hirsutum L.). Plant Cell Physiol. 51, 21-37

90 Rajhi, I. et al. (2011) Identification of genes expressed in maize root cortical cells during lysigenous aerenchyma formation using laser microdissection and microarray analyses. New Phytol. 190, 351-368

91 Steffens, B. and Sauter, M. (2009) Epidermal cell death in rice is confined to cells with a distinct molecular identity and is mediated by ethylene and $\mathrm{H}_{2} \mathrm{O}_{2}$ through an autoamplified signal pathway. Plant Cell 21, 184-196

92 Vidoz, M.L. et al. (2010) Hormonal interplay during adventitious root formation in flooded tomato plants. Plant J. 63, 551-562

93 de Guenni, L.B. et al. (2005) Regulation of natural hazards: floods and fires. In Ecosystems and Human Well-being: Current State and Trends (Vol. 1) (Hassan, R.M. et al., eds.), pp. 443-453, Millennium Ecosystem Assessment (Program), Condition and Trends Working Group, Island Press.

94 Jung, K.H. et al. (2010) The submergence tolerance regulator Sub1A mediates stress-responsive expression of AP2/ERF transcription factors. Plant Physiol. 152, 1674-1692

95 Seo, Y.S. et al. (2011) Towards establishment of a rice stress response interactome. PLoS Genet. 7, e1002020

96 Pena-Castro, J.M. et al. (2011) Expression of rice SUB1A and SUB1C transcription factors in Arabidopsis uncovers flowering inhibition as a submergence tolerance mechanism. Plant J. 67, 434-446 